\title{
Decentralized Traffic Control Using Agents in Ethernet Passive Optical Networks (EPON)
}

\author{
Sajaa K. Sadon*, Hizamel M. Hizan, Thavamaran Kanesan, Romli Mohamad \\ System Technologies Lab, Communication Technology, Telekom Research \& Development SdnBhd, \\ TMR \& D Innovation Centre, Lingkaran Teknokrat Timur, Cyberjaya, Malaysia \\ Email: "sajaa@tmrnd.com.my
}

Received 14 February 2016; accepted 23 August 2016; published 26 August 2016

Copyright (C) 2016 by authors and Scientific Research Publishing Inc.

This work is licensed under the Creative Commons Attribution International License (CC BY). http://creativecommons.org/licenses/by/4.0/

(c) (i) Open Access

\section{Abstract}

Traditionally, allocating of Ethernet Passive Optical Network (EPON) bandwidth schemes relied on a centralized architecture where their Optical Line Terminal (OLT) is the sole intelligent device with the capability for the arbitration of bandwidth based on time-division access for the upstream shared channel. However, any breakdown in the OLT bandwidth allocation will affect the allotment for Optical Network Units (ONUs). Few researches had dealt with a decentralized approach for the EPON and most of the solutions proposed involved additional cost by adding new complex devices to the original architecture. This paper proposes an intelligent decentralized mechanism in EPON that enhances the network performance using intelligent agents specification based on the IEEE 802.3 ah standards in the foundation for intelligent physical agent which is compatible with an Internet Protocol (IP)-based network. Specifically, this paper proposes a novel distributed scheme that supports differentiated services and ensures QoS for both Inter-and IntraONU allocation. The proposed mechanism introduces a unique direct communication between the ONUs supported by identical dynamic bandwidth allocation algorithms running simultaneously in each ONU. In addition to being fully distributed, the proposed scheme is asynchronous, scalable, and dynamic with flexibility and reliability in handling data, voice and video.

\section{Keywords}

Agents, Decentralized DBA, Diffserv, Distributed Systems, EPON

\footnotetext{
${ }^{*}$ Corresponding author.
}

How to cite this paper: Sadon, S.K., Hizan, H.M., Kanesan, T. and Mohamad, R. (2016) Decentralized Traffic Control Using Agents in Ethernet Passive Optical Networks (EPON). Communications and Network, 8, 193-203. 


\section{Introduction}

The increase in worldwide use of the Internet is expected to lead to increased options in distance learning, e-commerce, and communication that are multimedia-based. This trend will inevitably give rise to increased challenges in the organization of information and the need to facilitate greater retrieval efficiency. Greater demand for bandwidth requirements and managing of networks will be the new and additional challenges on top of the typical network connectivity problems from the network point of view. Therefore, a decentralized control is desirable to be tailored to the user's specific needs, e.g. emergence of voice, video and peer-to-peer applications $[1]$.

Currently, the centralized EPON bandwidth allocation schemes are prevalent where the traffic controller resides in the OLT. Thus, OLT has all the intelligences for the arbitration of time-division access to the shared channel.

In a distributed arbitration scheme, the ONUs themselves decide when to send data and for how long. In this scheme, prior to transmitting its data, all ONUs will transmit messages to announce the number of bytes to be sent. In the case of a round-robin arrangement, the ONU that is next on the schedule will track the transmission of the earlier ONU and monitor its transmission time to ensure that the transmission reaches the OLT soon after it is transmitted from the previous ONU. As such, a collision is avoided without wasting bandwidth [2]. Figure 1 shows the TDM-Bandwidth assignment for EPON.

Few researches have studied the decentralized approaches for the EPON. However, most of the solutions, as mentioned above, added cost and complexity to the original architecture proposed by IEEE 802.3 ah. It has been observed that decentralized EPON DBA algorithms can provide superior utilization of bandwidth but at a high cost [3].

The proposal of Sherif et al. [4] was for a new Ethernet over star coupler-based PON architecture that utilized the total distribution of a time-division multiple-access (TDMA) arbitration scheme. With the support of the dispersed or regional scheme, their study offered various QoS-based dynamic bandwidth allocation (DBA) algorithms that removed the OLT from the implementation of the time slot assignment.

Foh et al. [5] proposed an alternate solution for Ethernet passive optical networks. The solution uses a novel protocol named Full Utilization Local Loop (FULL) Request Contention Multiple Access (RCMA) protocol to efficiently provide communications in passive optical networks at the physical layer as well as the Media Access Control (MAC) layer protocol. The performance studies show that the MAC protocol is capable of offering $95 \%$ channel utilization under heavy load conditions.

However, with the expansion of the network span to $100 \mathrm{~km}$ and more, as recommended by next generation long-reach PONs (LR-PONs), the delays very significantly can erode the performance of these algorithms as their basis is bandwidth negotiation messages which are repeatedly communicated between the ONUs and the OLT.

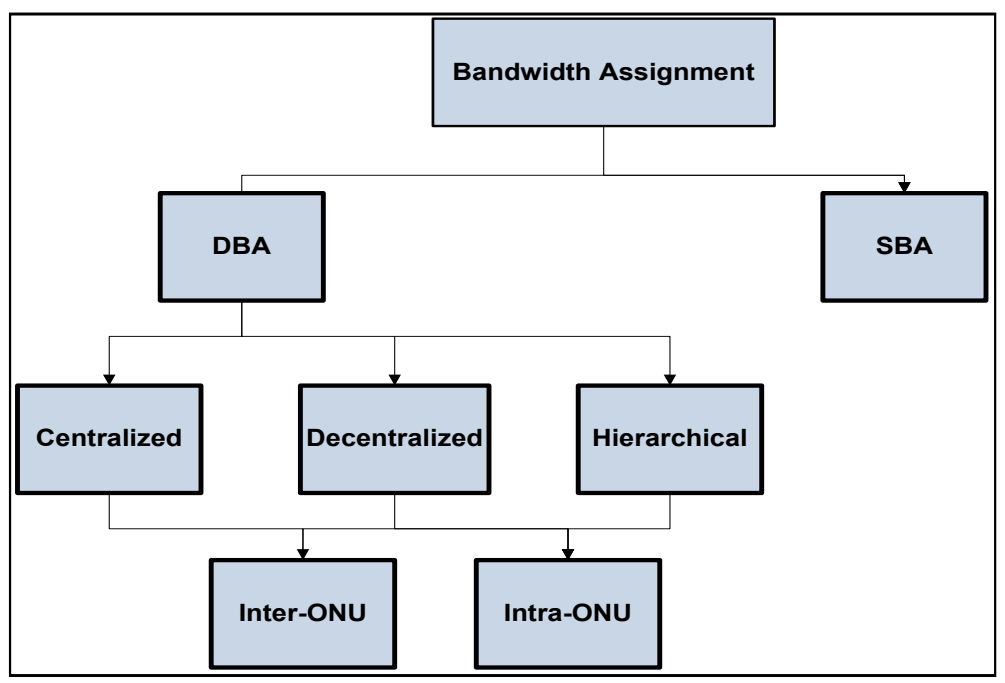

Figure 1. Agents communication messages. 
Feng et al. [6] published an article analyzing a distributed dynamic bandwidth allocation employing simulation. The algorithm used does not need any changes to the existing EPON frame, and only assigns the window information sent by other ONUs to a particular ONU through OLT. It is totally in accordance with the (Multi Pint Control Protocol) MPCP protocol of IEEE 802.3 ah standard and requires the addition of some new fields in the report and gate frames. However, this algorithm does not support traffic prioritization and thus provide no QoS guarantee.

Helmy et al. [7] proposed a regionalized media access scheme in the case of an emerging LR-PON that would accelerate transmission of upstream packets. They complement this scheme with centralized network control by using communication between ONUs on a control wavelength for the management of media access. When this wavelength is reflected back, it creates a multipoint-to-multipoint network among ONUs with the attachment of a Fiber Bragg Granting (FBG) to the splitter. This technique requires additional ONU transceivers even though they run at the rate of $100 \mathrm{Mbps}$.

Decentralized mechanism has been studied widely in different applications based on intelligent agents [8]. The notion behind any multi-agent system is to divide a complex problem handled by a single entry (a centralized system) into smaller simpler problems to be handled by various entities as in a distributed system.

In this study, an efficient decentralized allocation mechanism is introduced, which is critical to many technological domains such as traffic managements and bandwidth allocation for next generation access EPON networks. An agent refers to anything that can be observed as viewing its surroundings through sensors and reacting upon those surroundings via effectors. Eyes, rats and other organs are human agents and act as sensors, and hands, legs, mouth, and other body parts are effectors. In the case of robotic agent, cameras and infrared range finders act as sensors while some motors are the effectors. With software, the agent is in the form of encoded bit strings which are its preceptors and effectors [9].

The concept of agent is a crucial tool to create an open, heterogeneous and programmable network environment. This concept has been extensively proposed and widely accepted in the field of communications and Internet communities, motivated by the wish to employ the agents in the solution of problems that arise due to large-scale distribution and real time systems that involve the quantum and complexity of the tasks, latency, and delays among others.

In general, an agent is viewed as a complementary factor that is used to perform routine as well as complicated functions on behalf of the user. An agent in distributed computing is a part of independent software that acts in synch on behalf of the user and agents are either static or mobile. Most of the architecture of today's communication systems is of the client-server type requiring a multiplicity of transactions to accomplish a given task, and this implies additional signaling activity for the network, which is a challenge that can be escalated in the environment of an open network that covers multiple domains.

An agent can be defined as a piece of software that is capable of acting on behalf of others to accomplish a task. A more general description would state that an agent is a program authorized with some degree of autonomy to represent others in the same manner they would represent themselves or even better. The origin of agent research can be traced back to late 1976 when distributed artificial intelligence (DAI) was being initially studied. For more than a decade now, there has been much progress made in the research on software agents that emerged from work on artificial intelligence and distributed computing.

There can be another solution in mobile agents migrating the computations or interactions to the remote host by relocating the execution there. An example is mobile agents being deployed to perform their designated tasks independently, as long as a particular set of rules or constraints has been set specifically for them [10].

They can then be sent across the network as mobile programs or mobile codes that can be reassembled and performed in the remote host. In this work, agent technology is used primarily to automate the control and management processes in order to achieve greater network programmability for more rapid customization to provide new information and communications services.

It is also possible to use agents for the implementation of Service Level Agreements (SLAs) between different network actors and service era. For the implementation of SLA, an agent can be employed to mediate between users and service providers. Such an approach would simplify complex QoS metrics (from the view point of user). There can be negotiation between the agents of service provider and network provider and the agents of users to ensure the required service is met [11].

This paper proposes an intelligent decentralized direct communicability scheme between the ONUs-based EPON. It is a potential fiber-based communication because of its high speed, cost-effectiveness and scalability 
supported by a DBA algorithm based on intelligent agents' concepts, in which the bandwidth implementation assignment excludes the OLT. The proposed distributed EPON scheme is able to support the services that are differentiated and ensures QoS for both Inter and Intra ONU allocation.

\section{Direct Communication Using Agents}

The knowledge in software agents is becoming more important due to the dynamic and open environments in which they are able to interact by autonomously exchanging information and services with other software agents in a decentralized manner, thereby solving problems that cannot be solved by centralized systems [12]. For a distributed control plane to be implemented, direct connectivity (communicability) between the ONUs is required without imposing any restriction on the PON topology. By using the direct communicability scheme which employs agents, the ONUs swap and manage information pertaining to their queue status and their transmission needs among themselves by sending their request reports to the OLT. The distributed agents system will interchange all these information at once and each ONU will keep the other's queue status in a look-up table. A communication protocol based on software agents is implemented at the OLT and the ONUs. Following this, the ONUs simultaneously and autonomously run instances of the same DBA algorithm, providing identical bandwidth allocation results. When the algorithm is run, the ONUs in sequence and in an orderly manner send their data without any collisions, thus doing away with the need for the OLTs centralized task of processing the bandwidth allocation.

Previously, in order to ensure the synchronization in centralized arbitration schemes, it was necessary to determine the exact buffer state of a given ONU. Each ONU therefore has to lodge its bandwidth demand with the OLT prior to the OLT forwarding its request to the other ONUs [2].

Communication in a multi-agent system offers a means of swapping data on the basis of an acceptable set of rules or protocol of transmitting and receiving messages. On receipt of a message, an agent should have the ability to decipher what it means to send an appropriate response to create an intelligent structure of synchronization between agents to attain a common objective. An agent is required to communicate with other agents and to perform some actions for those agents [13].

\section{Proposed Intelligent Bandwidth Allocation}

The starting point of the simulation will begin by creating a chat between the agents. The dialog will contain an introduction of each Agent as location by getting the IP-address and marking the host with a specific number, so that they know each other in the system. Thus, a communication supported by communication language and a timer to ensure synchronization may allow exchanging and sharing of files and information between the agents.

The Intelligent agent inside the OLT assumes a cycle-based ACL (Agent Communication Language). The cycle is divided into three stages:

A. The first stage is used for synchronization and for sending traffic information as control messages; the OLT will send alleviate messages to each ONU telling the time slot specified for each ONU to send its queue status. Then each ONU employs its own fixed time slot in the update period to transmit its control message. To make it simple, and to eliminate collisions, these time slots are assigned according to a defined time-division multiple access (TDMA) assignment based on the fixed sizes of the control messages. It should be noted that the control slots in the proposed distributed scheme are all sent in sequence in one period (update period), which differs from. the centralized schemes mentioned before [14]-[19], where the control slot (REPORT Message) of each ONU is sent together with the data in the Transmission Window (TW) allocated to it by the OLT. All control update messages are transmitted as Ethernet frames.

B. The second stage of fixed length is a communication processing period which is utilized to allow the agents inside the ONUs to process the information collected from the multiplexed control message. Individual ONUs maintains an information table regarding the status of the queues at each other's ONU. At each cycle this information is updated or when there should be a new multiplexed control message received by an ONU from all other ONUs. The DBA algorithm module utilizes the table maintained at each ONU. Note that instances of the same DBA algorithm are executed simultaneously and autonomously at each ONU as shown in Figure 2, the conception design of the EPON system with decentralized DBA using Agents. Executing the algorithm results in a unique and identical set of ONU assignments; where there is the amount of bytes that an ONU may send in its TW during the cycle. The communication messages between the Agents will be as follows: 


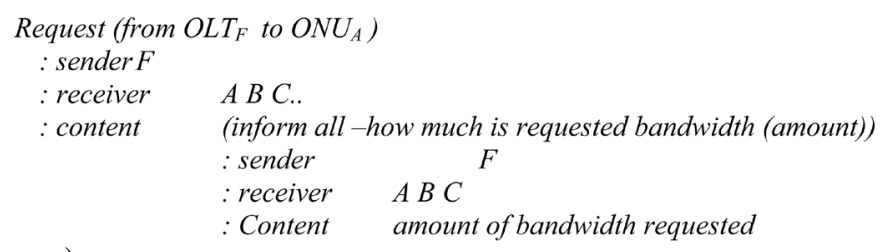

Agent $A B C$ inside each ONU will inform Agent $F$ inside $O L T$ about the requested bandwidth

(

inform

: sender $A B C$

$\therefore$ receiverF

: content requested bandwidth information

: reply-with: $\quad$ specific time)

)

Agent $F$ will broadcast the information to all ONUs to inform all about the requested bandwidth for the other ONUs

$\therefore$ sender $F$

: receiver

$A B C$.

: content

(inform all-

: sender

: receiver

- Content requested bandwidth for

)

Agents in ONUs inform Agent $F$ at the OLT about the allocation decision

(

inform

$\therefore$ sender $A B C$...

: receiver $\quad F$

: content allocation decision

)

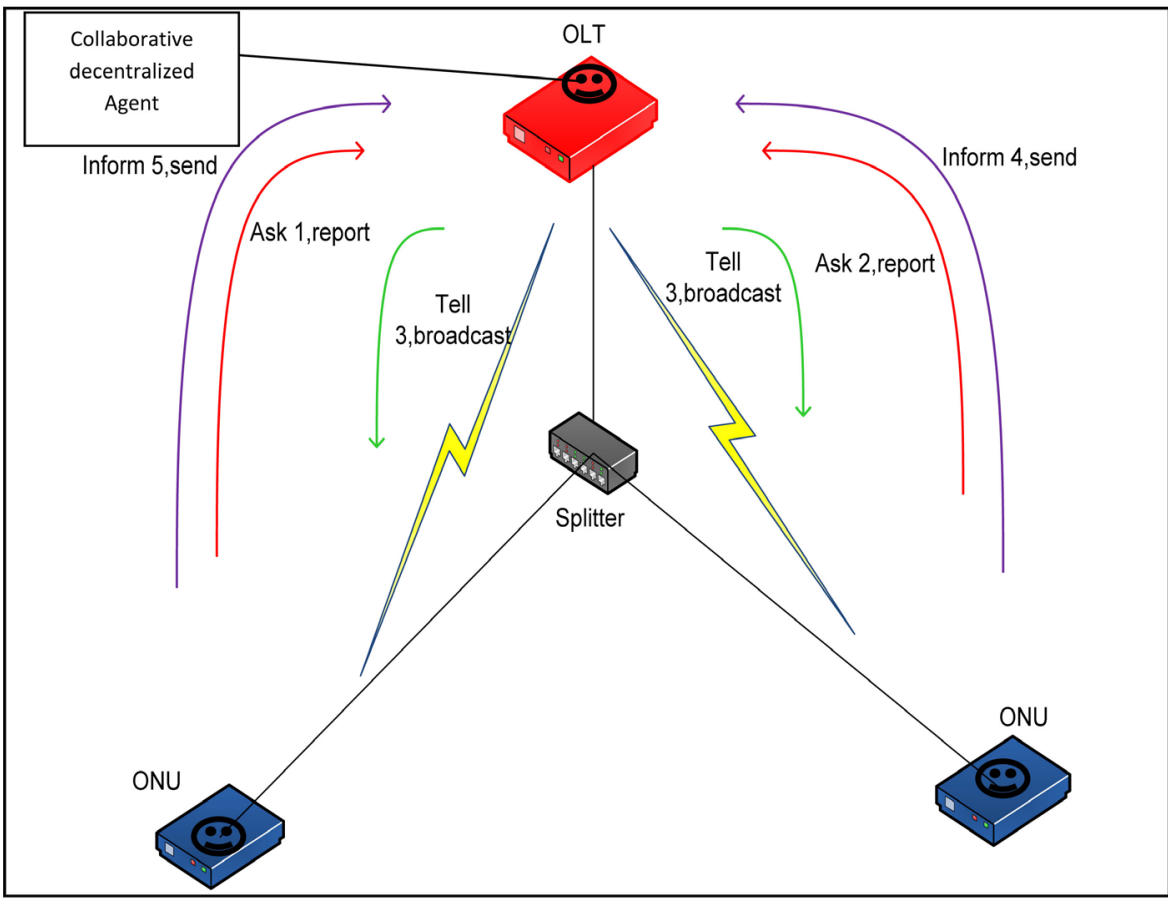

Figure 2. Intelligent Decentralized DBA flow. 
C. A dynamic allocation stage is utilized for the ONUs that follow exactly the allocation scheme produced by the algorithm based on Highest Priority Traffic First in descending order. Note that all ONUs will know when to start their transmissions, last for specific bytes and the order of ONUs transmission may vary for each cycle depending on the traffic status and not fixed [2] [19].

The Intelligent decentralized scheme possesses the additional flexibility of modifying the bandwidth allocated amount and sequence of ONUs transmission in line with the ONUs traffic demands, priority, and the extra bandwidth available. As the execution of the DBA and priority queuing scheduling tasks are both at the ONU, the DBA module can affect the integration of scheduling information to produce a globally optimized bandwidth allocation to a specific class of service in a given ONU. The ONUs transmission order will commence at the ONU with the highest priority traffic in descending order. The first transmission will be by the ONU with the highest bandwidth request.

Therefore, the order of ONUs transmission is not fixed and may vary in every cycle. As the DBA computation is running simultaneously and accordingly inside each ONU on the basis of the immediate global information sent via the Agents, if one or more ONU does not have much data to send and their request is less than the limited amount then the heavily loaded ONUs may be given the balance of the excessive bandwidth that is unused by the lightly loaded ONUs.

The decentralized algorithm will run simultaneously at each ONU, and the requested bandwidth for every ONU is then computed using the equation:

$$
R_{\text {onu }}=R_{\text {onu }}^{\mathrm{data}}+R_{\text {onu }}^{\mathrm{video}}+R_{\text {onu }}^{\mathrm{voice}}
$$

Equation (1) describes the summation of requested bandwidth for the three types of traffic; EF, AF and BE for each ONU. The value of rEF, rAF and rBE is achieved through assumption as according to the real traffic environment.When the bandwidth requested is less than the bandwidth allowed, what is provided is the requested bandwidth, based on the Differentiated Service (DiffServ) criteria, where the voice will get up to $20 \%$ of the available bandwidth with the highest priority, the video's portion is $40 \%$ with medium priority and the data's share is $40 \%$ with lowest priority.

If the total demanded bandwidth is higher than the available bandwidths then excessive bandwidth from other ONUs will be considered as shown in the equations below in the same way as the algorithm in [15].

$$
\begin{aligned}
& B_{\text {total }}^{\mathrm{demand}}=\sum_{\text {onu }=1}^{N} R_{\text {onu }}^{k}-\sum_{\text {onu }=1}^{N} B_{\text {onu }, k}^{\mathrm{min}} \\
& B_{\text {total }}^{\text {exces }}=\sum_{i=1}^{N} B_{\text {onu }, l}^{\mathrm{min}}-\sum_{\text {onu }}^{N} R_{\text {onu }}^{l}
\end{aligned}
$$

The ONUs will then be distributed the excess bandwidth as shown in the equation below: the ONUs use Equation (3) below [15], where $R_{\text {onu }}^{k}$ represents ONUs with heavy load, while $R_{\text {onu }}^{l}$ represents ONUs with light load.

$$
B_{\text {excess }}^{\text {onu }}=\frac{R_{\text {onu }}}{\sum_{K \in i} R_{\text {onu }}^{k}} * B_{\text {total }}^{\text {excess }} .
$$

To ensure the efficient utilization of excess bandwidth, the excessive bandwidth should be shared according to the requested bandwidth within the overloaded group.

$B_{\text {excess }}^{\text {onu }}$ is compared with the requested bandwidth, if it is higher than $R_{\text {onu }}$, then the bandwidth given will be only as much as requested. Therefore, no extra unused bandwidth will be wasted. The flow of the IDDBA algorithm is shown in Figure 3.

\section{Simulation Results and Discussion}

As a multiservice access network, the proposed architecture should support a multitude of services, i.e., triple play including best-effort data, video stream, and voice. In our simulation experiments, we classify our data into three priority classes: P0, P1, and P2 for delivering voice, video, and data traffic respectively. Expedited forwarding (EF) represents the delay sensitive real time service and the highest priority(voice) class, assured forwarding (AF) will be given to the real time with the less delay sensitive medium priority but assured bandwidth(video) class, and best-effort (BE) is for the lowest priority (data) class [20]-[22]. 


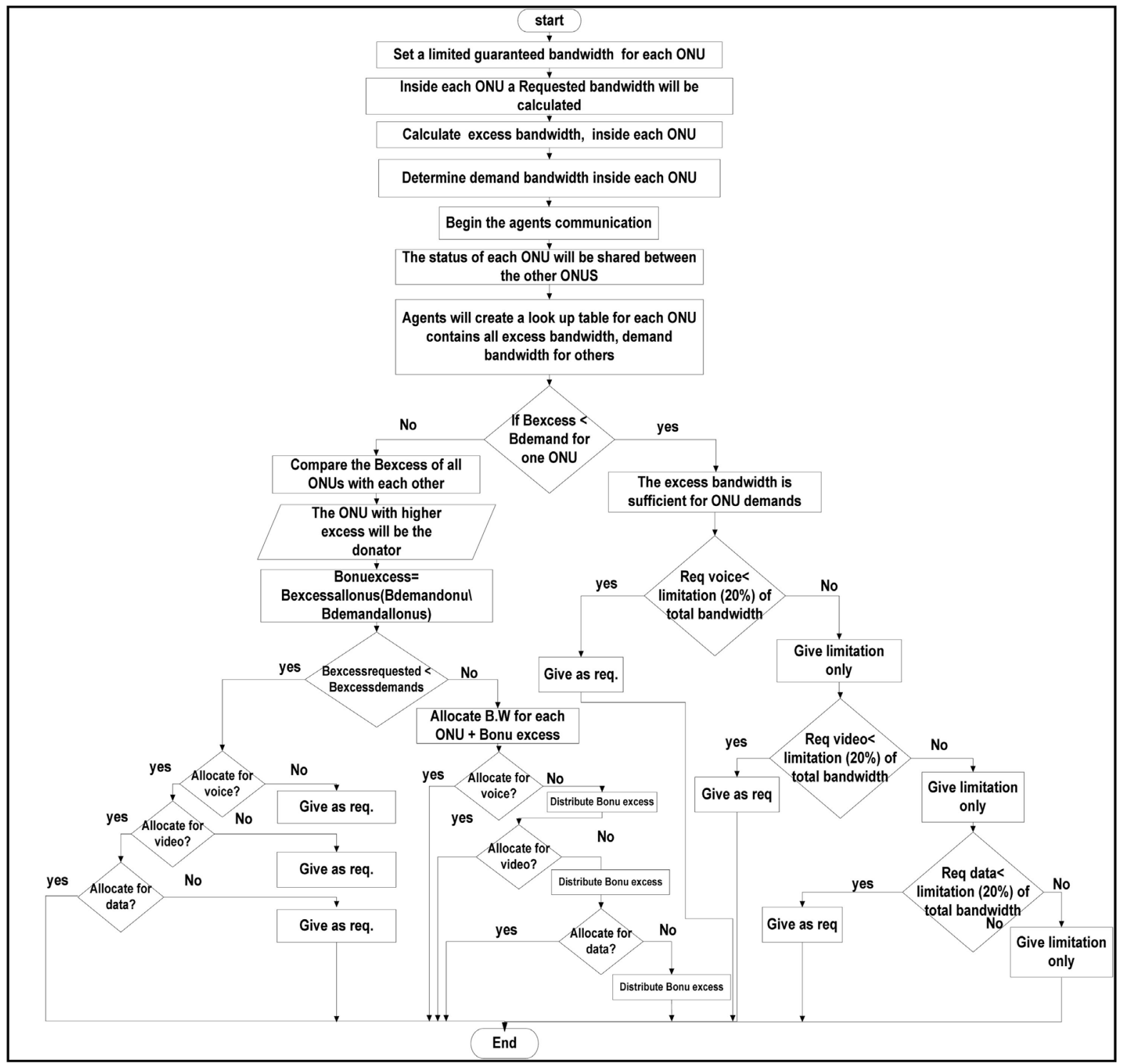

Figure 3. Intelligent Decentralized DBA flow.

The performance and the simulation parameters of the proposed IDDBA and previous DBA's procedures are shown in Table 1 and Table 2 . The proposed IDBA algorithm is designed to allocate the bandwidth in a decentralized manner and support QoS prospects. The interleaved polling with adaptive cycle tim (IPACT) [23] and Enhanced Decentralized DBA (EDDBA) [24].

The results based on fairness, bandwidth utilization and percentage of improvement are discussed below.

\section{A. Fairness Index}

Generally, the description of fairness is a parameter that states whether the bandwidth is justly shared between the ONUs. It varies between 0 and 1. The closer the fairness index to 1, the more evenly distributed the bandwidth. The result for fairness index is presented for the IDDBA and the previous algorithms are shown in Figure 4. The bandwidth is more evenly shared by IDDBA with a fairness value of almost 1 in contrast to other algorithms where the fairness index is lower especially at the high ONU load. This indicates almost equal distribution of bandwidth among the ONUs depending on their requested bandwidth. When the fairness index is 1 , the system is $100 \%$ fair. As the disparity increases, fairness decreases. $f$ in the context of the proposed algorithm in EPON is defined: 


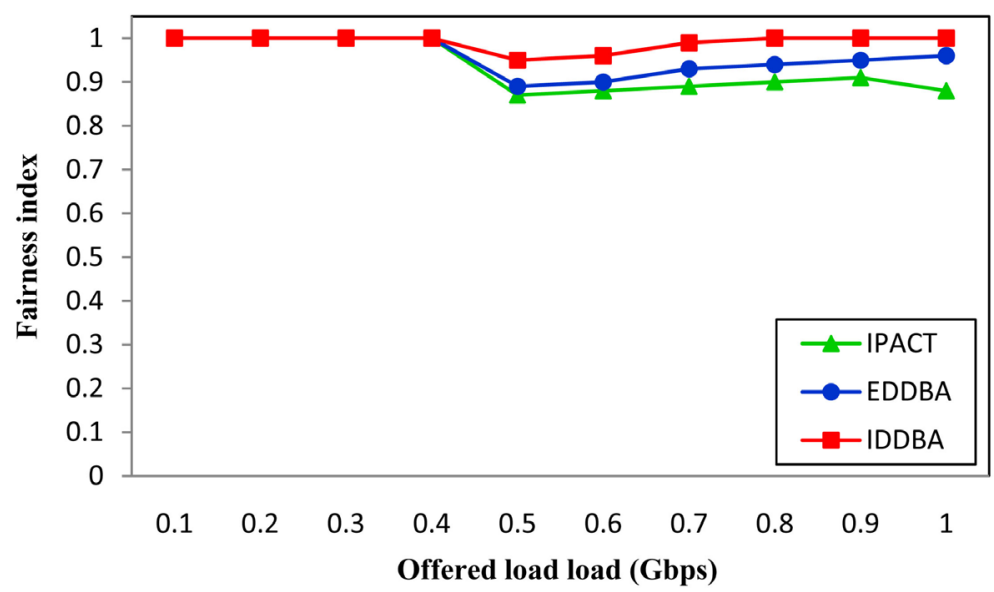

Figure 4. Bandwidth utilization versus offered load for IDDBA versus IPACT and EDDBA.

Table 1. Simulation parameters of proposed IDDBA with existing work.

\begin{tabular}{cccc}
\hline & & & Features \\
No & DBA algorithm & Advantages & Disadvantages \\
\cline { 3 - 4 } & IPACT & Inter-ONU scheduling & Doesn't support any QoS \\
2 & EDDBA & Inter \& Intra scheduling, good QoS & Still involve the OLT for bandwidth allocation \\
3 & IFLDBA & Inter \& Intra scheduling, good QoS, support A.I & Complex for testbed application \\
\hline
\end{tabular}

Table 2. Simulation parameters of proposed IDDBA with existing work.

\begin{tabular}{|c|c|c|c|}
\hline DBA & Simulation parameters & Working mechanism & Observation \\
\hline IPACT & $\begin{array}{c}\text { ONUs: } 8 \text {, total } \\
\text { bandwidth: } 1 \mathrm{G}, \\
\text { OLT-ONU distance: } 20 \mathrm{~km}\end{array}$ & $\begin{array}{l}\text { The OLT allocate bandwidth } \\
\text { for Inter \& Intra ONU }\end{array}$ & $\begin{array}{l}\text { Good performance, but it does not support } \\
\text { QoS and its centralized }\end{array}$ \\
\hline EDDBA & $\begin{array}{l}\text { ONUs: } 8 \text {, total bandwidth: } 1 \mathrm{G} \text {, } \\
\text { OLT-ONU distance: } 20 \mathrm{~km}\end{array}$ & $\begin{array}{l}\text { Each ONU allocate bandwidth } \\
\text { for its own through the OLT } \\
\text { broadcasted information }\end{array}$ & $\begin{array}{l}\text { Support QoS, but the decision not optimal efficient. } \\
\text { Decentralized, but still need the OLT arbitration }\end{array}$ \\
\hline IDDBA & $\begin{array}{l}\text { ONUs: } 8 \text {, total bandwidth: } 1 \mathrm{G} \text {, } \\
\text { OLT-ONU distance: } 20 \mathrm{~km}\end{array}$ & $\begin{array}{l}\text { The intelligent agent's } \\
\text { communication system provides } \\
\text { efficient sharing information } \\
\text { protocol between the ONUs }\end{array}$ & $\begin{array}{l}\text { Support QoS, but the decision is rational using agents } \\
\text { and the allocation impact is efficiently optimal. Fully } \\
\text { decentralized based on decentralized agent's machinery. } \\
\text { Shows good fairness due to collaborative behavior }\end{array}$ \\
\hline
\end{tabular}

$$
F=\frac{\left(\sum_{i=1}^{N} B_{i}\right)^{2}}{N\left(\sum_{i=1}^{N}\left(B_{i}\right)^{2}\right)}
$$

where $N$ is the total number of ONUs, $B_{i}$ is the allocated bandwidth for each ONU.

The three algorithms have almost similar performance in terms of fairness index for offered load 0 to 0.4 Gbps with a high fairness index due to low load applied. Beyond 0.4Gbps offered load, the three algorithms show a slightly less fairness between 0.4 and 0.6. This is due higher traffic data arrived to the network based on the bursty nature of EPON. However, IDDBA shows higher fairness index compared to EDDBA and IPACT, where it gets as high as 0.99 at full load, followed by EDDBA at 0.95 . While IPACT maintains to be the algorithm with the lowest fairness index where at full load, it has the fairness index is 0.84 .

\section{B. Bandwidth Utilization}


Consuming the bandwidth efficiently is a critical issue in order to make full use of the available resources and lower the cost of EPON-based access network. The operation of bandwidth utilization is shown in Figure 5, where, the description of bandwidth utilization in this paper is the amount of bandwidth allocated successfully depending on the requested bandwidth from the ONUs to OLT in a specific time period. Hence, the higher efficient usage of bandwidth offered, the better the performance of the EPON. In Figure 4, the bandwidth performances of the three algorithms are almost similar for ONUs load from 0 to $0.4 \mathrm{Gbps}$. At higher ONU load beyond $0.4 \mathrm{Gbps}$, IDDBA demonstrates the highest efficient bandwidth usage compared to previous algorithms, as the bandwidth utilization can be reached up to $89 \%$ which is $60 \%$ for EDDBA and $50 \%$ for IPACT at $1.1 \mathrm{Gbps}$.

\section{Percentage of improvement}

The improved percentage is defined as the gain obtained in the average allocated bandwidth of the proposed IDDBA algorithm compared to EDDBA and IPACT algorithms. The improvement of IPACT and EDDBA ranges between $3 \%$ up until $65 \%$ where the ONU load is 0.9 . Where $B_{I D B A}$ (for the new DBA) is the bandwidth received by the proposed DBA algorithm and $B_{\text {existing algorithm }}$ (comparison DBA) is the bandwidth received by the algorithms that are being compared as shown in Figure 6.

$$
I . P=\frac{B_{I D B A}-B_{\text {Existing } D B A}}{100} \times 100 \%
$$

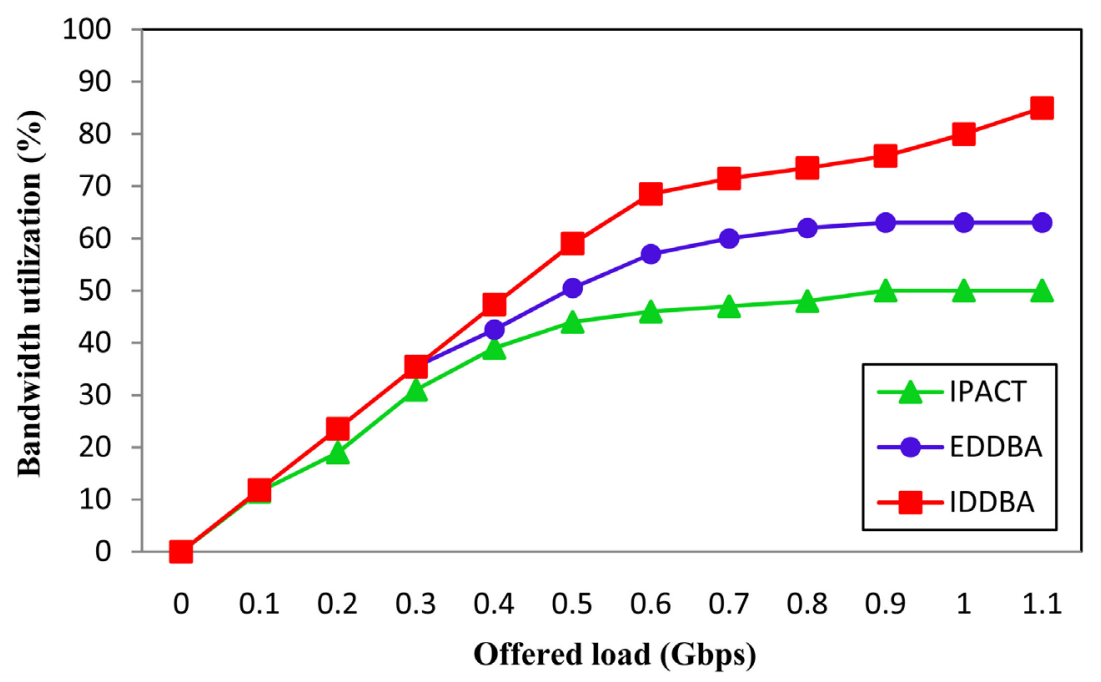

Figure 5. Bandwidth utilization versus offered load for IDDBA versus IPACT and EDDBA.

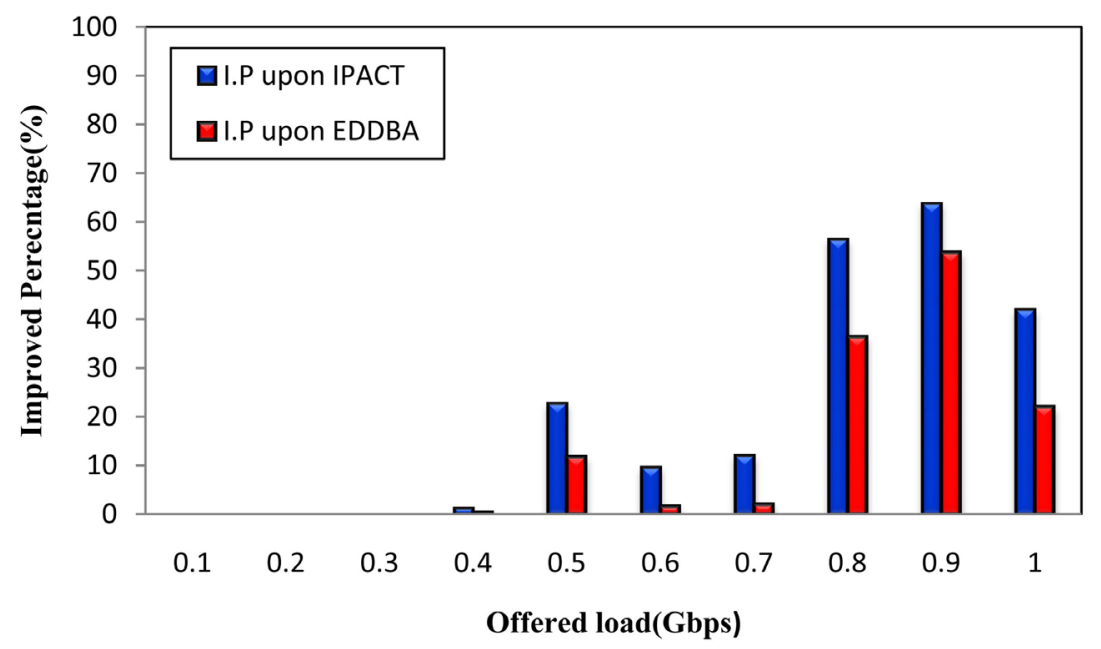

Figure 6. Improved percentage versus offered load for IDDBA upon IPACT and EDDBA. 


\section{Conclusion}

This article introduces the implementation of an EPON system supporting ONUs direct communication using IDDBA algorithm which ensures inter- and intra-ONU allocation algorithm independently at the ONU's side. The IDDBA supports triple play classes represented by voice, video, and data, and improves bandwidth efficiency by allowing the ONUs to share the uplink bandwidth in accordance with their bandwidth requests for various traffic priority classes autonomously. The decentralized IDDBA performance is analyzed to confirm the use of the bandwidth based on DiffServ functionalities with around $65 \%$ in contrast to IPACT and $25 \%$ compared to EDDBA.

\section{References}

[1] Le Faucher, F. (2005) Russian Dolls Bandwidth Constraints Model for Diffserv-Aware MPLS Traffic Engineering. IETF RFC 4127.

[2] Kramer, G. (2005) Ethernet Passive Optical Networks. McGraw-Hill Professional, ISBN: 0071445625.

[3] Maier, M. and Ghazisaidi, N. (2012) FiWi Access Networks. Cambridge University Press, Cambridge. www.cambridge.org

[4] Sherif, S.R., Hadjiantonis, A., Ellinas, G., Assi, C. and Ali, M.A. (2004) A Novel Decentralized Ethernet-Based PON Access Architecture for Provisioning Differentiated QOS. Journal of Light Wave Technology, 22, 2483-2497. http://dx.doi.org/10.1109/JLT.2004.836757

[5] Foh, C.H., Andrew, L., Wong, E. and Zukerman, M. (2004) FULL-RCMA: A High Utilization EPON. IEEE Journal on Selected Areas in Communications, 22, 1514-1524.

[6] Cao, F., Liu, D., Zhang, M., Yang, K. and Qian, Y. (2010) A Distributed Dynamic Bandwidth Allocation Algorithm in EPON. Modern Applied Science, 4, 20-24. http://dx.doi.org/10.5539/mas.v4n7p20

[7] Helmy, A.H., Fathallah, H. and Abdennour, A. (2011) Decentralized Media Access vs. Credit-Based Centralized Bandwidth Allocation for LR-PONs. High Capacity Optical Networks and Enabling Technologies, 2011, 329-333. http://dx.doi.org/10.1109/honet.2011.6149764

[8] Nallur, V. and Bahsoo, R. (2013) A Decentralized Self-Adaptation Mechanism for Service-Based Applications in the Cloud. IEEE Transactions on Software Engineering, 29, 591-612. http://dx.doi.org/10.1109/TSE.2012.53

[9] Nwana, H.S. (1996) Software Agents: An Overview. Intelligent Systems Research AT\&T, BT Laboratories Martlesham Heath, 11, 1-40.

[10] Agoulmine, N., Dragan, D., Gringel, T., Hall, J., Rosa, E. and Tschichholz, M. (2000) Trouble Management for Multimedia Services in Multi-Provider Environments. International Journal on Network and Service Management, 8, 99123.

[11] Hect-Nielsen, R. (1990) Neuro Computing. Addison-Wesley Publisher.

[12] Qureshi, M., Raza, A., Kumar, D., Yang, H.S. and Park, B.S. (2008) A Survey of Communication Network Paradigms for Substation Automation. IEEE International Symposium on Power Line Communications and Its Applications, 2008, 310-315. http://dx.doi.org/10.1109/ISPLC.2008.4510445

[13] Wooldridge, M.J. (1992) The Logical Modelling of Computational Multiagent Systems. PhD Thesis, University of Manchester, Manchester.

[14] Lung, B. (1999) PON Architecture "Future Proofs" FTTH. LightWave Magazine, 16, 104-107.

[15] Assi, C., Ye, Y., Dixit, S. and Ali, M.A. (2003) Dynamic Bandwidth Allocation for Quality of Service over Ethernet PONs. IEEE Journal on Selected Areas in Communications, 21, 1467-1477. http://dx.doi.org/10.1109/JSAC.2003.818837

[16] Sala, A. and Gummalla, A. (2001) PON Functional Requirements: Services and Performance. IEEE 802.3ah Meeting, Portland, July 2001.

[17] Kramer, G., Mukherjee, B. and Pesavento, G. (2001) Ethernet PON: Design and Analysis of an Optical Access Network. Photonic Network Communications, 3, 307-319. http://dx.doi.org/10.1023/A:1011463617631

[18] Kramer, G. and Pesavento, G. (2002) Ethernet Passive Optical Network (EPON): Building a Next Generation Optical Access Network. IEEE Communications Magazine, 40, 66-73. http://dx.doi.org/10.1109/35.983910

[19] Kramer, G., Mukherjee, B. and Pesavento, G. (2002) IPACT: A Dynamic Protocol for an Ethernet PON (EPON). IEEE Communications Magazine, 40, 74-80. http://dx.doi.org/10.1109/35.983911

[20] Ma, M. and Cheng, T.H. (2005) Adaptive Scheduling for Differentiated Services in an Ethernet Passive Optical Network. Journal of Optical Networking, 4, 661-670. http://dx.doi.org/10.1364/JON.4.000661 
[21] Min, L., Xiaomei, F., Yu, C. and Fulei, D. (2007) New Dynamic Bandwidth Allocation Algorithm for Ethernet PON. The 8th International Conference on Electronic Measurement and Instruments, Xi'an, July, 224-227.

[22] Moon, B. (2008) Emergency Handling in Ethernet Passive Optical Networks Using Priority-Based Dynamic Bandwidth Allocation. The 27th IEEE Conference on Computer Communications, Phoenix, 13-18 April 2008, 646-654. http://dx.doi.org/10.1109/infocom.2008.114

[23] Kramer, G., Mukherjee, B. and Pesavento, G. (2002) Interleaved Polling with Adaptive Cycle Time (IPACT): A Dynamic Bandwidth Distribution Scheme in an Optical Access Network. Photonic Network Communications, 4, 89-107. http://dx.doi.org/10.1023/A:1012959023043

[24] Sadon, S.K., Radzi, N.A., Din, N.M. and Al-Mansoori, M.H. (2013) Efficient Decentralized Dynamic Bandwidth Allocation in EPON. Proceedings of the World Congress on Engineering and Computer Science (WCECS), 2, 23-25.

\section{Submit or recommend next manuscript to SCIRP and we will provide best service for you:}

Accepting pre-submission inquiries through Email, Facebook, LinkedIn, Twitter, etc.

A wide selection of journals (inclusive of 9 subjects, more than 200 journals)

Providing 24-hour high-quality service

User-friendly online submission system

Fair and swift peer-review system

Efficient typesetting and proofreading procedure

Display of the result of downloads and visits, as well as the number of cited articles

Maximum dissemination of your research work

Submit your manuscript at: http://papersubmission.scirp.org/ 